\title{
HOMOTOPY DECOMPOSITIONS OF THE CLASSIFYING SPACES OF POINTED GAUGE GROUPS
}

\author{
STEPHEN THERIAULT
}

\begin{abstract}
Let $G$ be a topological group and let $\mathcal{G}^{*}(P)$ be the pointed gauge group of a principal $G$-bundle $P \longrightarrow M$. We prove that if $G$ is homotopy commutative then the homotopy type of the classifying space $B \mathcal{G}^{*}(P)$ can be completely determined for certain $M$. This also works $p$-locally, and valid choices of $M$ include closed simply-connected four-manifolds when localized at an odd prime $p$. In this case, an application is to calculate part of the mod- $p$ homology of the classifying space of the full gauge group.
\end{abstract}

\section{INTRODUCTION}

Let $G$ be a topological group and let $M$ be a pointed space. Let $P \longrightarrow M$ be a principal $G$-bundle over $M$. The gauge group $\mathcal{G}(P)$ is the group of $G$-equivariant automorphisms of $P$ that fix $M$. The pointed gauge group $\mathcal{G}^{*}(P)$ is the subgroup of $\mathcal{G}(P)$ that fixes the fibre over the basepoint in $M$. Gauge groups are of wide interest due to their prominent role in both mathematical physics, Donaldson theory, and the study of semi-stable holomorphic vector bundles and their related moduli spaces. Important problems are to calculate the mod- $p$ homology and cohomology of the classifying spaces $B \mathcal{G}(P)$ and $B \mathcal{G}^{*}(P)$ for a prime $p$ when $M$ is a closed simply-connected four-manifold, and to determine the integral homotopy types of various spaces related to $B \mathcal{G}^{*}(P)$ when $M$ is an orientable closed Riemann surface.

In this paper, assume that the topological groups have the homotopy type of connected, finite type $C W$-complexes. We show that if $G$ is homotopy commutative then for certain spaces $M$ there is a homotopy decomposition of $B \mathcal{G}^{*}(P)$ as recognizable factors. This also works $p$-locally. Two applications are given. The first is in the case when $G$ is a simply-connected, simple compact Lie group and $M$ is a closed simply-connected four-manifold. For appropriate primes $p$, a $p$-local homotopy decomposition of $B \mathcal{G}^{*}(P)$ holds and this is used to determine a large split subalgebra of the mod- $p$ cohomology of the full gauge group $B \mathcal{G}(P)$. The second is in the case when $G$ is the infinite unitary group and $M$ is a closed orientable Riemann surface. A homotopy decomposition of $B \mathcal{G}^{*}(P)$ is used to determine the homotopy type of the space $\operatorname{Hom}\left(\pi_{1}\left(\Sigma_{g}\right), U\right)$ of homorphisms from the fundamental group of the Riemann surface to the infinite unitary group.

2010 Mathematics Subject Classification. Primary 55P15, 55R35, Secondary 54C35, 81T13.

Key words and phrases. gauge group, mapping space, homotopy type, homology. 
The key result is a decomposition of certain pointed mapping spaces. Consider adjunction spaces of the form

$$
N=\left(\bigvee_{i=1}^{m} \Sigma A_{i}\right) \cup_{a} e^{n}
$$

where $\bigvee_{i=1}^{m} \Sigma A_{i}$ is a $C W$-complex of dimension strictly less than $n, a: S^{n-1} \longrightarrow \bigvee_{i=1}^{m} \Sigma A_{i}$ is the attaching map of the $n$-cell, and $m \geq 2$. For $1 \leq i \leq m$, let $\iota_{j}: \Sigma A_{j} \longrightarrow \bigvee_{i=1}^{m} \Sigma A_{i}$ be the inclusion of the $j^{t h}$-wedge summand. Let $\mathcal{N}$ be the collection of all such adjunction spaces $N$ with the additional property that the attaching map $a$ factors through a map $a^{\prime}$ which is a wedge sum of some of the Whitehead products $\Sigma A_{j} \wedge A_{k} \stackrel{\left[\iota_{j}, \iota_{k}\right]}{\longrightarrow} \bigvee_{i=1}^{m} \Sigma A_{i}$

Observe that there is a cofibration

$$
\bigvee_{i=1}^{m} \Sigma A_{i} \stackrel{b}{\longrightarrow} N \stackrel{q}{\longrightarrow} S^{n}
$$

where $b$ is the inclusion and $q$ collapses $\bigvee_{i=1}^{m} \Sigma A$ to a point. Let $G$ be a topological group and let $B G$ be its classifying space. Then the cofibration sequence induces a fibration sequence

$$
\operatorname{Map}^{*}(N, B G) \stackrel{b^{*}}{\longrightarrow} \operatorname{Map}^{*}\left(\bigvee_{i=1}^{m} \Sigma A_{i}, B G\right) \stackrel{a^{*}}{\longrightarrow} \operatorname{Map}^{*}\left(S^{n-1}, B G\right) .
$$

Theorem 1.1. Let $N \in \mathcal{N}$ and let $G$ be a topological group whose multiplication is homotopy commutative. Then the map $b^{*}$ in (1) has a right inverse and there is a homotopy equivalence

$$
\operatorname{Map}^{*}(N, B G) \simeq \operatorname{Map}^{*}\left(\bigvee_{i=1}^{m} \Sigma A_{i}, B G\right) \times \operatorname{Map}^{*}\left(S^{n}, B G\right) .
$$

A $p$-local version of Theorem 1.1 also holds if the multiplication on $G$ is only homotopy commutative at $p$. This is particularly relevant since James and Thomas [9] showed that no simplyconnnected, simple compact Lie group has its standard multiplication being homotopy commutative, but McGibbon [15] showed that after localizing at an odd prime there are cases when the multiplication is homotopy commutative and he classified these. The classification is given in Section 2 .

The connection with gauge groups comes from work of Gottlieb [5] or Atiyah and Bott [2]. They showed that if $M$ is a pointed space and $P \longrightarrow M$ is a principal $G$-bundle then there is a homotopy equivalence $B \mathcal{G}^{*}(P) \simeq \operatorname{Map}_{P}^{*}(M, B G)$, where $\operatorname{Map}_{P}^{*}(M, B G)$ is the component of $\operatorname{Map}^{*}(M, B G)$ that contains the map inducing $P$. Consider two cases. First, let $M$ be a closed simply-connected four-manifold and let $G$ be a simply-connected simple compact Lie group. By [16], $M$ is homotopy equivalent to a $C W$-complex $\left(\bigvee_{i=1}^{m} S^{2}\right) \cup_{a} e^{4}$. Second, let $M$ be an orientable closed Riemann surface of genus $g$ and let $G=U(n)$. Classically (see [6] for instance), $M$ is homotopy equivalent to a $C W$ complex $\left(\bigvee_{i=1}^{2 g} S^{1}\right) \cup_{a} e^{2}$. In either case, $[M, B G] \cong \mathbb{Z}$ so there is a component of $\operatorname{Map}^{*}(M, B G)$ for each integer $k$, and this integer determines a corresponding equivalence class of principal $G$-bundles $P \longrightarrow M$. Write $P_{k}$ for the equivalence class corresponding to $k$ and let $\mathcal{G}_{k}^{*}(M)=\mathcal{G}^{*}\left(P_{k}\right)$.

Let $\Omega_{0}^{3} G$ be the component of $\Omega^{3} G$ containing the basepoint. Write $X_{(p)}$ for a space $X$ localized at the prime $p$. 
Corollary 1.2. Let $M$ be a closed simply-connected Spin four-manifold with $m$ two-cells, $m \geq 2$, and let $G$ be a simply-connected simple compact Lie group whose multiplication is homotopy commutative when localized at $p$. Then there is a p-local homotopy equivalence

$$
B \mathcal{G}_{k}^{*}(M)_{(p)} \simeq\left(\prod_{i=1}^{m} \Omega G_{(p)}\right) \times \Omega_{0}^{3} G_{(p)} .
$$

In the second case, stabilize by considering the infinite unitary group $U$. Since $U$ is an infinite loop space its loop multiplication is homotopy commutative. Write $\Sigma_{g}$ for the surface of genus $g$, and let $\Omega_{0} U$ be the component of $\Omega U$ containing the basepoint.

Corollary 1.3. Let $\Sigma_{g}$ be a closed orientable closed Riemann surface of genus $g \geq 1$. Then there is an integral homotopy equivalence

$$
B \mathcal{G}_{k}^{*}\left(\Sigma_{g}\right) \simeq\left(\prod_{i=1}^{2 g} U\right) \times \Omega_{0} U
$$

Corollaries 1.2 and 1.3 are the first systematic decompositions of the classifying spaces of pointed gauge groups. In the context of Corollary 1.2, Masbaum [14] proved the $G=S U(2)$ case earlier but by using different methods that depended on the specific group. Also, while a great deal of work has been done recently to identify the $p$-local homotopy types of gauge groups $[10,12,13,19]$ and study their properties [11], nothing has been done for their classifying spaces.

Applications of these decompositions to the mod- $p$ homology of gauge groups and the homotopy type of $\operatorname{Hom}\left(\pi_{1}\left(\Sigma_{g}\right), U\right)$ will be discussed in the final section of the paper.

The author would like to thank the referee for making many valuable comments that helped improve the clarity of the paper.

\section{Preliminary homotopy theory}

In this section we discuss some notions from homotopy theory involving Whitehead products and the homotopy commutativity of topological groups. As we are building towards a strictly commutative diagram in (6) rather than a homotopy commutative diagram, some extra care will be taken along the way.

Let $G$ be a topological group and let

$$
e v: \Sigma \Omega B G \longrightarrow B G
$$

be the evaluation map. Let $i_{L}: \Sigma \Omega B G \longrightarrow \Sigma \Omega B G \vee \Sigma \Omega B G$ and $i_{R}: \Sigma \Omega B G \longrightarrow \Sigma \Omega B G \vee \Sigma \Omega B G$ be the inclusions of the left and right wedge summands respectively and let

$$
\left[i_{L}, i_{R}\right]: \Sigma \Omega B G \wedge \Omega B G \longrightarrow \Sigma \Omega B G \vee \Sigma \Omega B G
$$

be the Whitehead product of $i_{L}$ and $i_{R}$. By [1] there is a homotopy equivalence

$$
(\Sigma \Omega B G \vee \Sigma \Omega B G) \cup_{\left[i_{L}, i_{R}\right]} C(\Sigma \Omega B G \wedge \Omega B G) \simeq \Sigma \Omega B G \times \Sigma \Omega B G
$$


where $C(\Sigma \Omega B G \wedge \Omega B G)$ is the reduced cone on $\Sigma \Omega B G \wedge \Omega B G$. Let $t$ be the composite

$$
t: \Sigma \Omega B G \vee \Sigma \Omega B G \stackrel{e v \vee e v}{\longrightarrow} B G \vee B G \stackrel{\nabla}{\longrightarrow} B G
$$

where $\nabla$ is the folding map and let

$$
[e v, e v]: \Sigma \Omega B G \wedge \Omega B G \longrightarrow B G
$$

be the Whitehead product of $e v$ with itself. Note that $[e v, e v]$ is homotopic to $\nabla \circ\left[i_{L}, i_{R}\right]$. The following proposition connects the homotopy commutativity of $G$ to the existence of a certain extension.

Proposition 2.1. Let $G$ be a topological group. Then the following are equivalent:

(a) $G$ is homotopy commutative;

(b) the Whitehead product [ev, ev] is null homotopic;

(c) there is a strictly commutative diagram

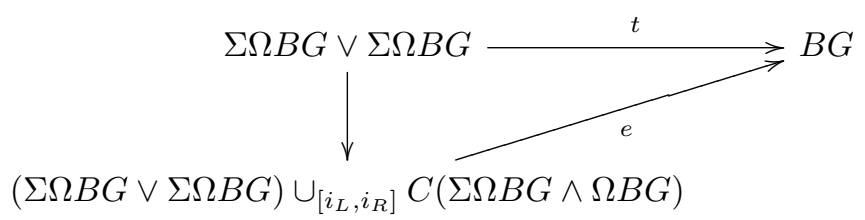

for some map e.

Proof. The equivalence of parts (a) and (b) was proved by James and Thomas [8] and the equivalence of parts (b) and (c) was proved by Arkowitz [1].

Remark 2.2. It should be noted that the homotopy commutativity condition in Proposition 2.1 is fairly restrictive. For example, there are no simply-connected, simple compact Lie groups which are homotopy commutative [9]. However, obstructions to homotopy commutativity may vanish when localized at a prime $p$ (see [7] for a good discussion of localization). McGibbon [15] classified those simply-connected, simple compact Lie groups $G$ which are homotopy commutative at $p$. To describe these, recall that $G$ is rationally homotopy equivalent to a product of spheres, $G \simeq_{\mathbb{Q}} \prod_{i=1}^{l} S^{2 n_{i}-1}$. The type of $G$ is defined to be $\left\{n_{1}, \ldots, n_{l}\right\}$. The loop multiplication on $G$ is homotopy commutative when localized at $p$ in precisely the following cases:

$$
p>2 n_{l} ; \quad G=S p(2) \text { and } p=3 ; \quad G=G_{2} \text { and } p=5 .
$$

On the other hand, Bott periodicity implies that the infinite matrix groups $U, S U, S O$ and $S p$ are all infinite loop spaces and so are integrally homotopy commutative. 
Next, we generalize the (a) implies (c) part of Proposition 2.1. Let $X_{1}, \ldots, X_{m}$ be path-connected, pointed spaces and consider the wedge $\bigvee_{i=1}^{m} \Sigma X_{i}$. For $1 \leq j \leq m$, let $\iota_{j}: \Sigma X_{j} \longrightarrow \bigvee_{i=1}^{m} \Sigma X_{i}$ be the inclusion of the $j^{\text {th }}$ wedge summand. Let

$$
f: \bigvee_{1 \leq j<k \leq m} \Sigma X_{j} \wedge X_{k} \longrightarrow \bigvee_{i=1}^{m} \Sigma X_{i}
$$

be the wedge sum of the Whitehead products $\left[\iota_{j}, \iota_{k}\right]$. Let

$$
T\left(\Sigma X_{1}, \ldots, \Sigma X_{m}\right)=\left(\bigvee_{i=1}^{m} \Sigma X_{i}\right) \cup_{f} C\left(\bigvee_{1 \leq j<k \leq m} \Sigma X_{j} \wedge X_{k}\right)
$$

Observe that there is a homotopy equivalence

$$
T\left(\Sigma X_{1}, \ldots, \Sigma X_{m}\right) \simeq \bigcup_{1 \leq j<k \leq m} \Sigma X_{j} \times \Sigma X_{k}
$$

To be clear, $T\left(\Sigma X_{1}, \ldots, \Sigma X_{m}\right)$ is a subspace of $\Sigma X_{1} \times \cdots \times \Sigma X_{m}$, each $\Sigma X_{j} \times \Sigma X_{k}$ in the union is regarded as including into the $(j, k)$ coordinates of $\Sigma X_{1} \times \cdots \times \Sigma X_{m}$, and intersections are identified.

This construction is natural. Suppose that there are maps $g: \Sigma A \longrightarrow Z, h: \Sigma B \longrightarrow Z$ and $t: Z \longrightarrow Z^{\prime}$. Represent the homotopy class $[g, h]$ as the adjoint of the Samelson product $\left\langle g^{\prime}, h^{\prime}\right\rangle$, where $g^{\prime}: A \longrightarrow \Omega Z$ and $h^{\prime}: B \longrightarrow \Omega Z$ are the adjoints of $g$ and $h$ respectively. The Samelson product is defined by the pointwise commutator in $\Omega Z$, which commutes with any loop map $\Omega Z \stackrel{\Omega t}{\longrightarrow}$ $\Omega Z^{\prime}$. Thus we obtain $t \circ[g, h]=[t \circ g, t \circ h]$ on the nose. Hence, given maps $f_{i}: \Sigma X_{i} \longrightarrow \Sigma X_{i}^{\prime}$ for $1 \leq i \leq m$, we obtain a strictly commutative diagram

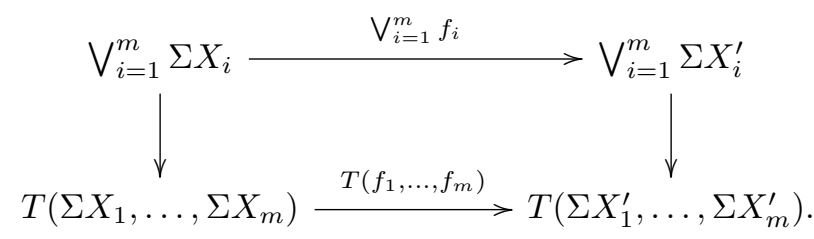

In our case, for $1 \leq i \leq m$, let $X_{i}=\Omega B G$. Write $T(\Sigma \Omega B G)$ for $T(\Sigma \Omega B G, \ldots, \Sigma \Omega B G)$. Let $t_{m}$ be the composite

$$
t_{m}: \bigvee_{i=1}^{m} \Sigma \Omega B G \stackrel{\bigvee_{i=1}^{m} \text { ev }}{\longrightarrow} \bigvee_{i=1}^{m} B G \stackrel{\nabla_{m}}{\longrightarrow} B G
$$

where $\nabla_{m}$ is the $m$-fold folding map. By Proposition 2.1, if $G$ is homotopy commutative then the restriction of $t_{m}$ to any pair $\Sigma \Omega B G \vee \Sigma \Omega B G$ extends to a map

$$
(\Sigma \Omega B G \vee \Sigma \Omega B G) \cup_{\left[i_{L}, i_{R}\right]} C(\Sigma \Omega B G \wedge \Omega B G) \longrightarrow B G
$$

Construct an extension for all pairs of wedge summands indexed by $(j, k)$ for $1 \leq j<k \leq m$. Observe that the extensions are compatible because they intersect only on the wedge summands. Thus they may be assembled to produce a map $T(\Sigma \Omega B G) \longrightarrow B G$ extending $t_{m}$. This is recorded as follows. 
Lemma 2.3. Let $G$ be a topological group whose loop multiplication is homotopy commutative, Then there is a strictly commutative diagram

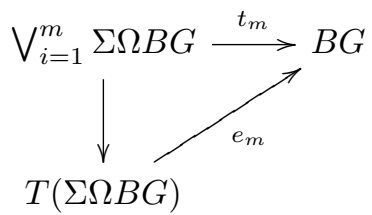

for some map $e_{m}$.

We close this section with one more observation about $T\left(\Sigma X_{1}, \ldots, \Sigma X_{m}\right)$. Let $X \stackrel{E}{\longrightarrow} \Omega \Sigma X$ be the suspension map, defined by sending $x \in X$ to the loop $\omega_{x}$ on $\Sigma X$, where $\omega_{x}$ is characterized by $\omega_{x}(t)=(t, x)$. The evaluation map $\Sigma \Omega Y \stackrel{e v}{\longrightarrow} Y$ is defined by sending $(s, \omega)$ to $\omega(s)$. The definitions imply that the composite $\Sigma X \stackrel{\Sigma E}{\longrightarrow} \Sigma \Omega \Sigma X \stackrel{e v}{\longrightarrow} \Sigma X$ is the identity map on $\Sigma X$. Now suppose that there is a map $f: \Sigma X \longrightarrow Y$. The naturality of the evaluation map implies that there is a strictly commutative diagram

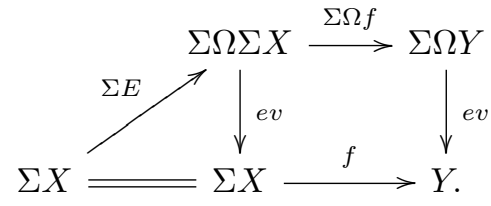

Thus, if $\bar{f}=(\Sigma \Omega f) \circ \Sigma E$, then we obtain a lift

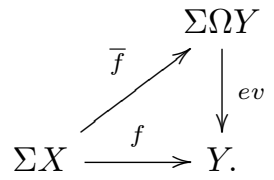

Combining this with (3) we obtain the following.

Lemma 2.4. Suppose that for $1 \leq i \leq m$ there are maps $f_{i}: \Sigma X_{i} \longrightarrow Y$. Then there is a strictly commutative diagram

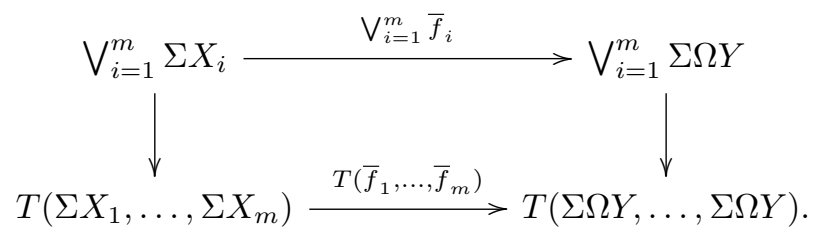

\section{THE CLASS $\mathcal{N}$}

Recall from the Introduction that $\mathcal{N}$ is the class of adjunction spaces

$$
N=\left(\bigvee_{i=1}^{m} \Sigma A_{i}\right) \cup_{a} e^{n}
$$

where $\bigvee_{i=1}^{m} \Sigma A_{i}$ is a $C W$-complex of dimension strictly less than $n$, the attaching map $a$ factors through a map $a^{\prime}$ which is a wedge sum of some of the Whitehead products $\Sigma A_{j} \wedge A_{k} \stackrel{\left[\iota_{j}, \iota_{k}\right]}{\longrightarrow}$ 
$\bigvee_{i=1}^{m} \Sigma A_{i}$, and $m \geq 2$. The factorization condition on $a$ can be restrictive. In the context of gauge groups, one typically wants to work with an $N$ that is homotopy equivalent to a manifold. Most manifolds do not satisfy the attaching map condition. However, there are some very interesting families of manifolds that do. For example:

(1) if $M$ is a simply-connected Spin 4-manifold with $H^{2}(M ; \mathbb{Z})$ of rank $m \geq 2$ then $M$ is homotopy equivalent to a $C W$-complex $\left(\bigvee_{i=1}^{m} S^{2}\right) \cup_{a} e^{4} \in \mathcal{N}$;

(2) if $\Sigma_{g}$ is a closed orientable surface of genus $g \geq 1$ then $\Sigma_{g}$ is homotopy equivalent to a $C W$-complex $\left(\bigvee_{i=1}^{2 g} S^{1}\right) \cup_{a} e^{2} \in \mathcal{N}$

(3) if $M$ is a simply-connected Spin 5-manifold then $M$ is homotopy equivalent to a $C W$-complex $\left(\bigvee_{i=1}^{m} \Sigma A_{i}\right) \cup_{a} e^{5}$ where each $\Sigma A_{i}$ is either $S^{2}, S^{3}$ or a mod- $p^{r}$ Moore space of dimension 3, and if $m \geq 2$ then this $C W$-complex is in $\mathcal{N}$.

The $C W$-structure for $M$ in (1) is due to Milnor [16]; the $C W$-structure for $\Sigma_{g}$ in (2) is commonly known, one reference is [6]; the $C W$-structure for $M$ in (3) is given in [18]. Other examples exist, such as certain $(n-1)$-connected $2 n$-dimensional manifolds [20] and the connected sum of products of two spheres.

The property that is needed for the spaces in $\mathcal{N}$ is the following. Recall that there is a homotopy cofibration $S^{n-1} \stackrel{a}{\longrightarrow} \bigvee_{i=1}^{m} \Sigma A_{i} \stackrel{b}{\longrightarrow} N$ where $b$ is the inclusion.

Lemma 3.1. Let $N \in \mathcal{N}$. Then there is an extension

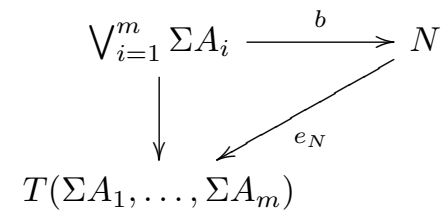

for some map $e_{N}$.

Proof. Since $N=\left(\bigvee_{i=1}^{m} \Sigma A_{i}\right) \cup_{a} e^{n}$, to show that the extension $e_{N}$ exists it is equivalent to show that the composite $S^{n-1} \stackrel{a}{\longrightarrow} \bigvee_{i=1}^{m} \Sigma A_{i} \longrightarrow T\left(\Sigma A_{1}, \ldots, \Sigma A_{m}\right)$ is null homotopic. By definition, $T\left(\Sigma A_{1}, \ldots, \Sigma A_{m}\right)$ is the adjunction space formed from coning off the sum of all the Whitehead products $\left[\iota_{j}, \iota_{k}\right]$ for $1 \leq j<k \leq m$. In particular, each composition $\Sigma A_{j} \wedge A_{k} \stackrel{\left[\iota_{j}, \iota_{k}\right]}{\longrightarrow} \bigvee_{i=1}^{m} \Sigma A_{i} \longrightarrow$ $T\left(\Sigma A_{1}, \ldots, \Sigma A_{m}\right)$ is null homotopic. Thus, as a factors through a wedge sum of some of the Whitehead products $\left[\iota_{j}, \iota_{k}\right]$, the composite $S^{n-1} \stackrel{a}{\longrightarrow} \bigvee_{i=1}^{m} \Sigma A_{i} \longrightarrow T\left(\Sigma A_{1}, \ldots, \Sigma A_{m}\right)$ is also null homotopic.

\section{A decomposition of $\operatorname{MaP}^{*}(N, B G)$}

Let $N \in \mathcal{N}$. In the sequence of maps

$$
S^{n-1} \stackrel{a}{\longrightarrow} \bigvee_{i=1}^{m} \Sigma A_{i} \stackrel{b}{\longrightarrow} N \stackrel{q}{\longrightarrow} S^{n}
$$


the maps $a$ and $b$ form a homotopy cofibre sequence, while $b$ and $q$ form a cofibre sequence on the nose. If $G$ is a topological group then there is an induced sequence

$$
\operatorname{Map}^{*}\left(S^{n}, B G\right) \stackrel{q^{*}}{\longrightarrow} \operatorname{Map}^{*}(N, B G) \stackrel{b^{*}}{\longrightarrow} \operatorname{Map}^{*}\left(\bigvee_{i=1}^{m} \Sigma A_{i}, B G\right) \stackrel{a^{*}}{\longrightarrow} \operatorname{Map}^{*}\left(S^{n-1}, B G\right)
$$

where the maps $q^{*}$ and $b^{*}$ form a fibre sequence on the nose while $b^{*}$ and $a^{*}$ form a homotopy fibre sequence. We will show that if the multiplication on $G$ is homotopy commutative then the map $b^{*}$ has a right inverse.

Let $f: \bigvee_{i=1}^{m} \Sigma A_{i} \longrightarrow B G$ be a pointed map. Universally, a map out of a wedge is determined by its restrictions to the wedge summands, so $f=\bigvee_{i=1}^{m} f_{i}$ where $f_{i}: \Sigma A_{i} \longrightarrow B G$ is the restriction of $f$ to $\Sigma A_{i}$. By (4), each $f_{i}$ lifts through $\Sigma \Omega B G \stackrel{e v}{\longrightarrow} B G$ to a map $\bar{f}_{i}=\left(\Sigma \Omega f_{i}\right) \circ \Sigma E$. So if $N \in \mathcal{N}$ and the multiplication on $G$ is homotopy commutative, we may combine the diagrams in Lemmas 2.3, 2.4 and 3.1 to obtain a strictly commutative diagram

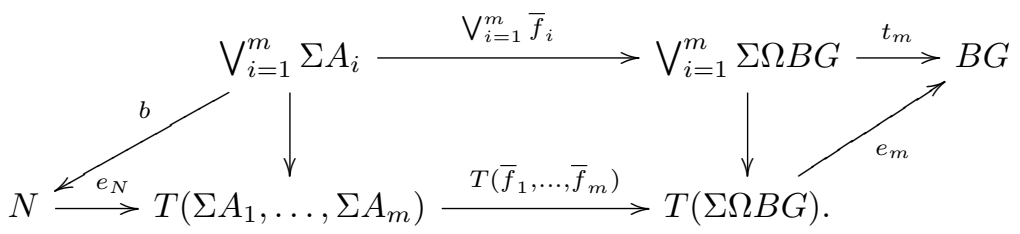

By the definitions of $t_{m}$ and each $\bar{f}_{i}$, we have $t_{m} \circ\left(\bigvee_{i=1}^{m} \bar{f}_{i}\right)=\bigvee_{i=1}^{m} f_{i}$. So (6) lets us define a map

$$
\theta: \operatorname{Map}^{*}\left(\bigvee_{i=1}^{m} \Sigma A_{i}, B G\right) \longrightarrow \operatorname{Map}^{*}(N, B G)
$$

by $\theta(f)=\theta\left(\bigvee_{i=1}^{m} f_{i}\right)=e_{m} \circ T\left(\bar{f}_{1}, \ldots, \bar{f}_{m}\right) \circ e_{N}$. We wish to show that $\theta$ is continuous and that $b^{*} \circ \theta$ is the identity map.

Lemma 4.1. The map $\theta$ is continuous.

Proof. The map $\theta$ is defined as the composite of the continuous maps $e_{m}$ and $e_{N}$ and the continuous functor $T\left(\bar{f}_{1}, \ldots, \bar{f}_{m}\right)$. Note that if $Y$ is a locally compact Hausdorff space then the composition $\operatorname{Map}^{*}(Y, Z) \times \operatorname{Map}^{*}(X, Y) \longrightarrow \operatorname{Map}^{*}(X, Z)$ is continuous with respect to the compact open topology. Therefore $\theta$ is continuous.

Lemma 4.2. The composite of continuous maps

$$
\operatorname{Map}^{*}\left(\bigvee_{i=1}^{m} \Sigma A_{i}, B G\right) \stackrel{\theta}{\longrightarrow} \operatorname{Map}^{*}(N, B G) \stackrel{b^{*}}{\longrightarrow} \operatorname{Map}^{*}\left(\bigvee_{i=1}^{m} \Sigma A_{i}, B G\right)
$$

is equal to the identity map.

Proof. By definition, $b^{*}$ sends a map $\phi: N \longrightarrow B G$ to the composite $\bigvee_{i=1}^{m} \Sigma A_{i} \stackrel{b}{\longrightarrow} N \stackrel{\phi}{\longrightarrow} B G$. Therefore, by definition of $\theta$, we have

$$
b^{*} \circ \theta(f)=b^{*} \circ \theta\left(\bigvee_{i=1}^{m} f_{i}\right)=b^{*}\left(e_{m} \circ T\left(\bar{f}_{1}, \ldots, \bar{f}_{m}\right) \circ e_{N}\right)=e_{m} \circ T\left(\bar{f}_{1}, \ldots, \bar{f}_{m}\right) \circ e_{N} \circ b .
$$


By (6) and the definition of $t_{m}$, we have

$$
e_{m} \circ T\left(\bar{f}_{1}, \ldots, \bar{f}_{m}\right) \circ e_{N} \circ b=t_{m} \circ\left(\bigvee_{i=1}^{m} \bar{f}_{i}\right)=\bigvee_{i=1}^{m} f_{i}=f .
$$

Thus $b^{*} \circ \theta(f)=f$.

Proof of Theorem 1.1. In general, suppose that $\Omega B \stackrel{\partial}{\longrightarrow} F \stackrel{r}{\longrightarrow} E \stackrel{s}{\longrightarrow} B$ is a homotopy fibration sequence and $r$ has a right homotopy inverse $t: E \longrightarrow F$. Then $s$ is null homotopic because: (i) $r \circ t \simeq 1_{E}$ implies that $s \simeq s \circ r \circ t$, and (ii) $s \circ r$ is null homotopic as it is the composition of two consecutive maps in a homotopy fibration. The null homotopy for $s$ implies that $F \simeq E \times \Omega B$. In our case, consider the homotopy fibration sequence (5). By Lemma 4.2, the map $b^{*}$ has a right inverse. Therefore there is a homotopy equivalence

$$
\operatorname{Map}^{*}(N, B G) \simeq \operatorname{Map}^{*}\left(\bigvee_{i=1}^{m} \Sigma A_{i}, B G\right) \times \operatorname{Map}^{*}\left(S^{n}, B G\right) \text {. }
$$

To illustrate Theorem 1.1 we consider two cases of interest. Note that $\operatorname{Map}^{*}\left(S^{t}, B G\right) \simeq \Omega^{t-1} G$.

Example 4.3. Let $M$ be a simply-connected Spin 4-manifold with $m$ two-cells, where $m \geq 2$. As in Section 3, there is a homotopy equivalence $M \simeq\left(\bigvee_{i=1}^{m} S^{2}\right) \cup_{a} e^{4}$. Let $G$ be a simply-connected, simple compact Lie group listed in (2), whose multiplication is homotopy commutative when localized at $p$. By [7], p-localization commutes with mapping spaces in the context of simply-connected (and more generally, nilpotent) spaces, so we have $\operatorname{Map}^{*}(M, B G)_{(p)} \simeq \operatorname{Map}^{*}\left(M_{(p)}, B G_{(p)}\right)$. Thus Theorem 1.1 implies that there is a homotopy equivalence

$$
\operatorname{Map}^{*}(M, B G)_{(p)} \simeq\left(\prod_{i=1}^{m} \Omega G_{(p)}\right) \times \Omega^{3} G_{(p)} .
$$

Example 4.4. Let $\Sigma_{g}$ be a close orientable surface of genus $g \geq 1$. As in Section $3, \Sigma_{g} \simeq$ $\left(\bigvee_{i=1}^{2 g} S^{1}\right) \cup_{a} e^{2} \in \mathcal{N}$. Let $G=U$, the infinite unitary group. Since $U$ is an infinite loop space it is homotopy commutative so by Theorem 1.1 there is a homotopy equivalence

$$
\operatorname{Map}^{*}\left(\Sigma_{g}, B U\right) \simeq\left(\prod_{i=1}^{2 g} U\right) \times \Omega U
$$

We close this section by proving Corollaries 1.2 and 1.3 .

Proof of Corollary 1.2. Recall from the Introduction that if $G$ is a simply-connected simple compact Lie group, $M$ is a simply-connected four-manifold and $P_{k} \longrightarrow M$ is a principal $G$-bundle induced by the homotopy class in $[M, B G] \cong \mathbb{Z}$ corresponding to $k$, then there is a homotopy equivalence $B \mathcal{G}_{k}^{*}(M) \simeq \operatorname{Map}_{k}^{*}(M, B G)$. By Example 4.3, there is a $p$-local homotopy equivalence $\operatorname{Map}_{k}^{*}(M, B G)_{(p)} \simeq\left(\prod_{i=1}^{m} \Omega G_{(p)}\right) \times \Omega_{k}^{3} G_{(p)}$, where $\Omega_{k}^{3} G$ is the connected component of $\Omega^{3} G$ that contains the map $S^{3} \longrightarrow G$ of degree $k$ in the third homology group. Since $\pi_{0}\left(\Omega^{3} G\right)$ is a group, 
there is a homotopy equivalence $\Omega_{k}^{3} G \simeq \Omega_{0}^{3} G$. Therefore $B \mathcal{G}_{k}^{*}(M)_{(p)} \simeq\left(\prod_{i=1}^{m} \Omega G_{(p)}\right) \times \Omega_{0}^{3} G_{(p)}$, as asserted.

Proof of Corollary 1.3. Again, recall from the Introduction that if $G=U, \Sigma_{g}$ is a closed orientable surface of genus $g$ and $P_{k} \longrightarrow \Sigma_{g}$ is a principal $G$-bundle induced by the homotopy class in $\left[\Sigma_{g}, B U\right] \cong \mathbb{Z}$ corresponding to $k$, then there is a homotopy equivalence $B \mathcal{G}_{k}\left(\Sigma_{g}\right) \simeq \operatorname{Map}_{k}^{*}\left(\Sigma_{g}, B U\right)$. By Example 4.4, there is a homotopy equivalence $\operatorname{Map}_{k}^{*}\left(\Sigma_{g}, B U\right) \simeq\left(\prod_{i=1}^{2 g} U\right) \times \Omega_{k} U$ where $\Omega_{k} U$ is the connected component of $\Omega U$ that contains the map $S^{1} \longrightarrow U$ of degree $k$ in the first homology group. Since $\pi_{0}(\Omega U)$ is a group, there is a homotopy equivalence $\Omega_{k} U \simeq \Omega_{0} U$. Therefore there is a homotopy equivalence $B \mathcal{G}_{k}\left(\Sigma_{g}\right) \simeq\left(\prod_{i=1}^{2 g} U\right) \times \Omega U$, as asserted.

\section{Applications}

In this section we give two applications, one to the calculation of the mod- $p$ homology or cohomology of the classifying space of certain full gauge groups, and the other to the homotopy type of a certain group of homomorphisms.

First, return to the case when $G$ is a simply-connected simple compact Lie group, $M$ is a simplyconnected four-manifold, and $P_{k} \longrightarrow M$ is a principal $G$-bundle induced by the homotopy class in $[M, B G] \cong \mathbb{Z}$ corresponding to $k$. By $[2]$ there is a homotopy commutative diagram

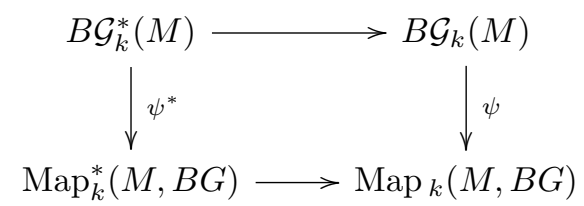

where $\psi^{*}$ and $\psi$ are homotopy equivalences. Observe also that there is a fibration

$$
\operatorname{Map}_{k}^{*}(M, B G) \longrightarrow \operatorname{Map}_{k}(M, B G) \stackrel{e v}{\longrightarrow} B G
$$

where $e v$ evaluates a map at the basepoint of $M$. Stated in terms of gauge groups, up to homotopy equivalences, there is a fibration

$$
B \mathcal{G}_{k}^{*}(M) \longrightarrow B \mathcal{G}_{k}(M) \longrightarrow B G
$$

Take homology and cohomology with mod- $p$ coefficients. Corollary 1.2 immediately implies that if $G$ is homotopy commutative when localized at $p$ then there is a coalgebra isomorphism

$$
H_{*}\left(B \mathcal{G}_{k}^{*}(M)\right) \cong\left(\otimes_{i=1}^{m} H_{*}(\Omega G)\right) \otimes H_{*}\left(\Omega_{0}^{2} G\right)
$$

and an algebra isomorphism

$$
H^{*}\left(B \mathcal{G}_{k}^{*}(M)\right) \cong\left(\otimes_{i=1}^{m} H^{*}(\Omega G)\right) \otimes H^{*}\left(\Omega_{0}^{2} G\right)
$$

We aim to prove the following. 
Theorem 5.1. Let $M$ be a closed simply-connected Spin four-manifold and let $G$ be a simplyconnected simple compact Lie group whose multiplication is homotopy commutative when localized at $p$. Then the composite of coalgebras

$$
\otimes_{i=1}^{m} H_{*}(\Omega G) \longrightarrow H_{*}\left(B \mathcal{G}_{k}^{*}(M)\right) \longrightarrow H_{*}\left(B \mathcal{G}_{k}(M)\right)
$$

has a left inverse, and the composite of algebras

$$
H^{*}\left(B \mathcal{G}_{k}(M)\right) \longrightarrow H^{*}\left(B \mathcal{G}_{k}^{*}(M)\right) \longrightarrow \otimes_{i=1}^{m} H^{*}(\Omega G)
$$

has a right inverse.

For example, let $G=S U(2)$, in which case $G$ is homeomorphic to $S^{3}$ and $H^{*}\left(\Omega S^{3}\right)$ is well known. This case is of key interest in Donaldson theory and a major open problem is to calculate the mod- $p$ homology of $B \mathcal{G}_{k}(M)$. As $S U(2)$ is homotopy commutative when localized at primes $p \geq 5$, Theorem 5.1 applies for any such prime, giving significant information about $H_{*}\left(B \mathcal{G}_{k}(M)\right)$.

To prove Theorem 5.1, we begin by recalling some general facts about mapping spaces. Let $X_{1}, \ldots, X_{m}$ and $Y$ be Hausdorff spaces, and let $\coprod_{i=1}^{m} X_{i}$ be their disjoint union. Then there is a homemorphism

$$
\operatorname{Map}\left(\coprod_{i=1}^{m} X_{i}, Y\right) \cong \prod_{i=1}^{m} \operatorname{Map}\left(X_{i}, Y\right) .
$$

Further, if each of $X_{1}, \ldots, X_{m}$ and $Y$ are pointed, then there is a homeomorphism

$$
\operatorname{Map}^{*}\left(\bigvee_{i=1}^{m} X_{i}, Y\right) \cong \prod_{i=1}^{m} \operatorname{Map}^{*}\left(X_{i}, Y\right) \text {. }
$$

These two decompositions are compatible in the following sense. There is a quotient map

$$
\mathfrak{q}: \coprod_{i=1}^{m} X_{i} \longrightarrow \bigvee_{i=1}^{m} X_{i}
$$

which identifies the basepoints in each space $X_{i}$ to a common point. So there is an induced map

$$
\mathfrak{q}^{*}: \operatorname{Map}\left(\bigvee_{i=1}^{m} X_{i}, Y\right) \longrightarrow \operatorname{Map}\left(\coprod_{i=1}^{m} X_{i}, Y\right) \text {. }
$$

The two homeomorphisms above are compatible via a strictly commutative diagram

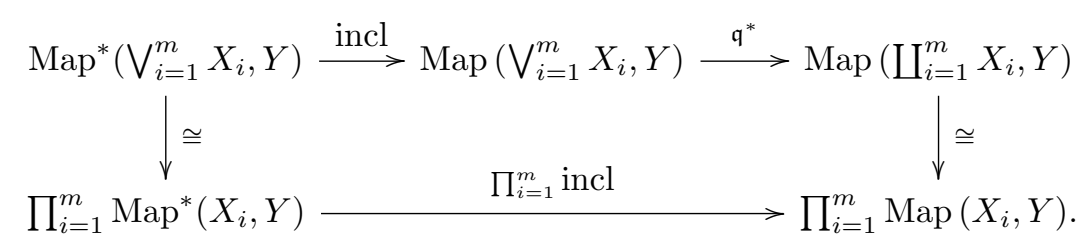

Returning to the case of interest, as in Section 3, if $M$ is any closed simply-connected Spin 4manifold then there is a space $N=\left(\bigvee_{i=1}^{m} S^{2}\right) \cup_{a} e^{4} \in \mathcal{N}$. The inclusion $\bigvee_{i=1}^{m} S^{2} \stackrel{b}{\longrightarrow} N$ induces a 
commutative diagram

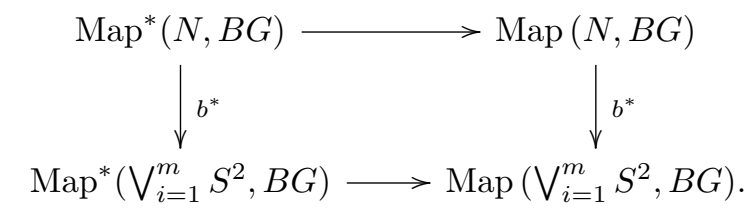

Localizing at $p$, the fact that mapping spaces commute with localization of nilpotent spaces [7] implies that there is a homotopy commutative diagram

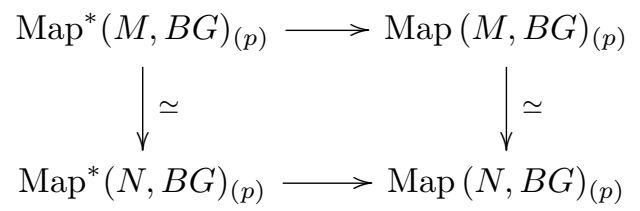

Juxtaposing the diagrams (7), (8), (9) and (10) we obtain a p-local homotopy commutative diagram

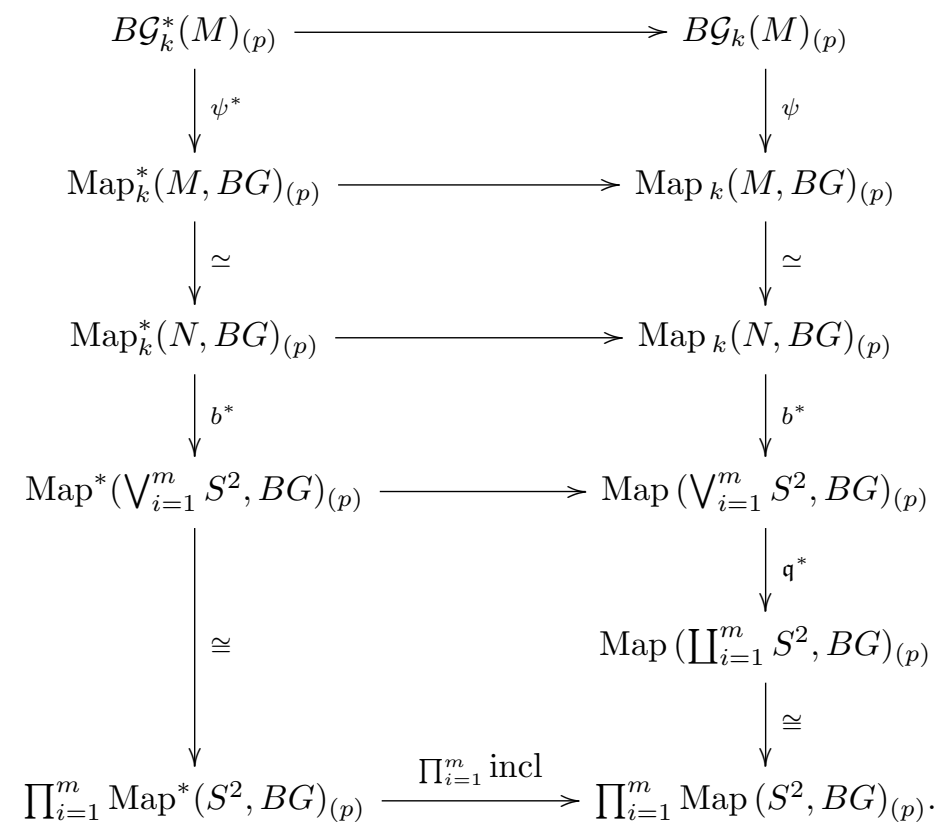

By Lemma 4.2, the map $b^{*}$ has a right inverse. Lifting this, up to homotopy, through the homotopy equivalences $B \mathcal{G}_{k}^{*}(M)_{(p)} \stackrel{\psi^{*}}{\longrightarrow} \operatorname{Map}_{k}^{*}(M, B G)_{(p)} \stackrel{\simeq}{\longrightarrow} \operatorname{Map}_{k}^{*}(N, B G)_{(p)}$, we obtain the following.

Lemma 5.2. Let $M$ be a closed simply-connected Spin four-manifold and let $G$ be a simply-connected simple compact Lie group whose multiplication is homotopy commutative when localized at a prime $p$. Then there is a homotopy commutative diagram

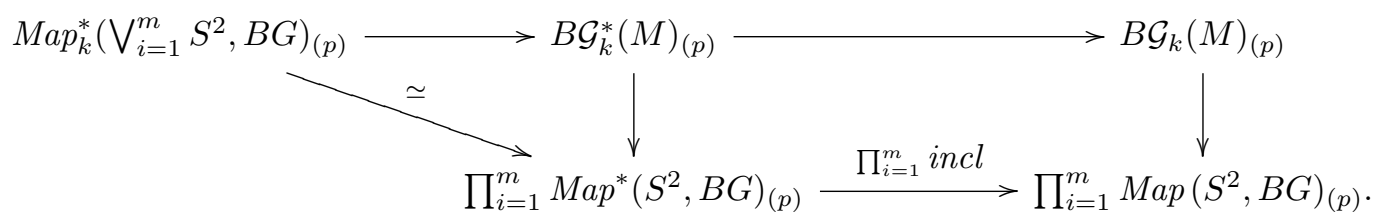

Lemma 5.2 is used to extract information about $H_{*}\left(B \mathcal{G}_{k}(M)\right)$ and $H^{*}\left(B \mathcal{G}_{k}(M)\right)$. 
Proof of Theorem 5.1. Consider the map $\operatorname{Map}^{*}\left(S^{2}, B G\right) \stackrel{\text { incl }}{\longrightarrow} \operatorname{Map}\left(S^{2}, B G\right)$ whose $p$-localization appears in the bottom row of the diagram in Lemma 5.2. The inclusion is the fibre of the evaluation map $\operatorname{Map}\left(S^{2}, B G\right) \stackrel{e v}{\longrightarrow} B G$ which sends a map $f: S^{2} \longrightarrow B G$ to $f(*)$. Also, we have $\operatorname{Map}^{*}\left(S^{2}, B G\right)=\Omega G$. So there is a fibration

$$
\Omega G \longrightarrow \operatorname{Map}\left(S^{2}, B G\right) \stackrel{e v}{\longrightarrow} B G
$$

By (2), the cases when the multiplication on $G$ is homotopy commutative when localized at $p$ are known. In each such case, $H^{*}(G)$ is an exterior algebra on odd degree generators, so by [3] $H^{*}(B G)$ is a polynomial algebra on even degree generators. Since cohomology is with mod-p coefficients, we can dualize to see that $H_{*}(B G)$ is also concentrated in even degrees. Further, by [4] the integral cohomology of $\Omega G$ is concentrated in even degrees, and therefore so is the mod- $p$ cohomology. Therefore the homology Serre spectral sequence for the fibration (11) collapses at the $E^{2}$-term and there are no extension issues. Hence

$$
H_{*}\left(\operatorname{Map}\left(S^{2}, B G\right)\right) \cong H_{*}(B G) \otimes H_{*}(\Omega G)
$$

Consequently, taking homology for the diagram in Lemma 5.2, we see that the composite

$$
\otimes_{i=1}^{m} H_{*}(\Omega G) \longrightarrow H_{*}\left(B \mathcal{G}_{k}^{*}(M)\right) \longrightarrow H_{*}\left(B \mathcal{G}_{k}(M)\right)
$$

has a left inverse.

Similarly,

$$
H^{*}\left(\operatorname{Map}\left(S^{2}, B G\right)\right) \cong H^{*}(B G) \otimes H^{*}(\Omega G)
$$

and the composite

$$
H^{*}\left(B \mathcal{G}_{k}(M)\right) \longrightarrow H^{*}\left(B \mathcal{G}_{k}^{*}(M)\right) \longrightarrow \otimes_{i=1}^{m} H^{*}(\Omega G)
$$

has a right inverse.

We now turn to the second application. Let $K$ and $L$ be topological groups, and let $\operatorname{Hom}(K, L)$ be the set of homomorphisms from $K$ to $L$, topologized as a subspace of the mapping space $\operatorname{Map}(K, L)$. If $B K, B L$ are the classifying spaces of $K$ and $L$ respectively, there is a natural map

$$
B: \operatorname{Hom}(K, L) \longrightarrow \operatorname{Map}^{*}(B K, B L)
$$

This map has been a subject of intense study due to its connections with the Sullivan conjecture in homotopy theory, to the moduli space of representations in algebraic geometry, and to the space of flat connections modulo gauge equivalence in Yang-Mills theory. Consider the special case

$$
\operatorname{Hom}\left(\pi_{1}\left(\Sigma_{g}\right), U(n)\right) \longrightarrow \operatorname{Map}^{*}\left(B \pi_{1}\left(\Sigma_{g}\right), B U(n)\right)
$$


Since the universal cover of $\Sigma_{g}$ is contractible there is a homotopy equivalence $\Sigma_{g} \simeq B \pi_{1}\left(\Sigma_{g}\right)$. So up to a homotopy equivalence we may regard the preceding map as

$$
\operatorname{Hom}\left(\pi_{1}\left(\Sigma_{g}\right), U(n)\right) \longrightarrow \operatorname{Map}^{*}\left(\Sigma_{g}, B U(n)\right)
$$

Ramras [17, Theorem 3.4] used gauge theoretic methods to show that this map is an injection on $\pi_{0}$ and an isomorphism on $\pi_{m}$ for $m \leq 2 g(n-1)+1$. Stabilizing to the infinite unitary group, we obtain a map

$$
\operatorname{Hom}\left(\pi_{1}\left(\Sigma_{g}\right), U\right) \longrightarrow \operatorname{Map}^{*}\left(\Sigma_{g}, B U\right)
$$

which is an injection on $\pi_{0}$ and an isomorphism on $\pi_{m}$ for every $m \geq 1$. Thus if $\left.\operatorname{Hom}_{I}\left(\pi_{1}\left(\Sigma_{g}\right), U\right)\right)$ is the component of $\left.\operatorname{Hom}\left(\pi_{1}\left(\Sigma_{g}\right), U\right)\right)$ containing the identity map, from Corollary 1.3 we obtain homotopy equivalences

$$
\left.\operatorname{Hom}_{I}\left(\pi_{1}\left(\Sigma_{g}\right), U\right)\right) \stackrel{\simeq}{\longrightarrow} \operatorname{Map}_{0}^{*}\left(\Sigma_{g}, B U\right) \stackrel{\simeq}{\longrightarrow}\left(\prod_{i=1}^{2 g} U\right) \times \Omega_{0} U
$$

which lets one easily identify $\pi_{m}\left(\operatorname{Hom}\left(\pi_{1}\left(\Sigma_{g}\right), U\right)\right)$ for $m \geq 1$.

\section{REFERENCES}

1. M. Arkowitz, The generalized Whitehead product, Pacific J. Math. 12 (1962), 7-23.

2. M.F. Atiyah and R. Bott, The Yang-Mills equations over Riemann surfaces, Philos. Trans. Roy. Soc. London Ser. A 308 (1983), 523-615.

3. A. Borel, Sur la cohomologie des espaces fibrés prinicpaux et des espaces homogènes de groupes de Lie compacts, Ann. of Math. 57 (1953), 115-207.

4. R. Bott, An application of Morse theory to the topology of Lie groups, Bull. Soc. Math. France 84 (1956), 251-281.

5. D.H. Gottlieb, Applicatios of bundle map theory, Trans. Amer. Math. Soc. 171 (1972), 23-50.

6. A. Hatcher, Algebraic Topology, Cambridge University Press, Cambridge, 2002.

7. P. Hilton, G. Mislin and J. Roitberg, Localization of nilpotent groups and spaces, North-Holland Mathematical Studies 15, North-Holland Publishing, Amsterdam-Oxford, 1975.

8. I.M. James and E. Thomas, On homotopy-commutativity, Ann. of Math. 76 (1962), 9-17.

9. I.M. James and E. Thomas, Homotopy-abelian topological groups, Topology 1 (1962), 237-240.

10. D. Kishimoto and A. Kono, Splitting of gauge groups, Trans. Amer. Math. Soc. 362 (2010), 6715-6731.

11. D. Kishimoto, A. Kono and S. Theriault, Homotopy commutativity in p-localized gauge groups, Proc. Roy. Soc. Edin. 143 (2013), 851-870.

12. D. Kishimoto, A. Kono and S. Theriault, Refined decompositions of gauge groups, to appear in Kyoto J. Math.

13. D. Kishimoto, A. Kono and M. Tsutaya, Mod-p decompositions of gauge groups, Algebr. Geom. Topol. 13 (2013), $1757-1778$

14. G. Masbaum, On the cohomology of the classifying space of the gauge group over some 4-complexes, Bull. Soc. Math. France 119 (1991), 1-31.

15. C.A. McGibbon, Homotopy commutativity in localized groups, Amer. J. Math 106 (1984), 665-687.

16. J. Milnor, On simply-connected 4-manifolds, 1958 symposium internacional de topología algebraica International symposium on algebraic topology, pp. 122-128, Universidad Nacional Autónoma de México and UNESCO, Mexico City. 
17. D.A. Ramras, The stable moduli space of flat connections over a surface, Trans. Amer. Math. Soc. 363 (2011), 1061-1100

18. R. Stöcker, On the structure of 5-dimensional Poincaré duality spaces, Comment. Math. Helv. 57 (1982), $481-510$.

19. S.D. Theriault, Odd primary homotopy decompositions of gauge groups, Algebr. Geom. Topol. 10 (2010), 535-564.

20. C.T.C Wall, Classification of $(n-1)$-connected $2 n$-manifolds, Ann. of Math. 75 (1962), 163-189.

Mathematical Sciences, University of Southampton, Southampton SO17 1BJ, United Kingdom

E-mail address: S.D.Theriault@soton.ac.uk 